\title{
Impact of incorporations of various polysaccharides on rheological and microstructural characteristics of heat-induced quinoa protein isolate gels
}

\author{
Shubham Patole ${ }^{1} \cdot$ Lirong Cheng $^{2} \cdot$ Zhi Yang $^{1}$ (D)
}

Received: 15 November 2021 / Accepted: 27 January 2022 / Published online: 22 February 2022

(c) The Author(s)

\begin{abstract}
This study aimed to investigate the properties of heat-induced gels $\left(85^{\circ} \mathrm{C}\right.$ for $\left.30 \mathrm{~min}\right)$ of quinoa protein isolate (QPI) in the presence and absence of various polysaccharides including guar gum (GG), locust bean gum (LBG), and xanthan gum (XG) at $\mathrm{pH}$ 7. For this purpose, samples with three gum concentrations $(0.05,0.1$, and $0.2 \mathrm{wt} \%)$ at a fixed QPI concentration $(10$ $\mathrm{wt} \%)$ and a fixed ionic strength $(50 \mathrm{mM} \mathrm{NaCl})$ were studied in terms of their gelation behaviour, small and large deformation rheological properties, water holding capabilities, and microstructural characteristics. Rheological measurements revealed that all polysaccharides incorporation could improve gel strength (complex modulus, $G^{*}$ ) and breaking stress, accelerate gel formations, and more stiffer gels were obtained at greater polysaccharide concentrations. The XG exhibited the most gel strengthening effect followed by LBG and GG. Incorporation of $0.2 \mathrm{wt} \% \mathrm{XG}$ led to a 15 folds increase in $G^{*}$ compared to the control. Confocal laser scanning microscopy observation revealed that the polysaccharides also altered gel microstructures, with the gels containing XG showing the most compact gel structures. The findings of this study may provide useful information for the fabrication of novel QPI based food gel products with improved texture.
\end{abstract}

Keywords Quinoa protein isolates · Polysaccharides · Viscoelasticity $\cdot$ Gelation $\cdot$ Microstructure

\section{Introduction}

Animal proteins have been dominated in the Western diet for a long time, as they have a high amino acid score, easier digestibility, and greater water solubility for the required nutrition of humans [1]. However, meat production has a significant impact on environment and contributes to greenhouse gas emission [2]. Making food production more sustainable in order to feed fast growing population, one solution is to increasingly investigate and utilize novel plant protein sources and decrease the consumption of animal-based food [3]. Recently, extensive efforts have been devoted to developing plant protein based food such as meat substitutes, infant formulae, and dairy alternatives, and some of them still commercially available [4-6].

Zhi Yang

z.yang2@massey.ac.nz

1 School of Food and Advanced Technology, Massey University, Auckland 0632, New Zealand

2 The Riddet Institute, Palmerston North 4472, New Zealand
Quinoa (Chenopodium quinoa) is a pseudocereal and being an important protein and nutritional resource for the people from its origin, South America [7]. The total protein content in quinoa seeds varies from 12 to $23 \%$ (based on dry weight) depending on variety and origin [7] . Quinoa protein isolates (QPI) have a significant amount of all essential amino acids, and it is mainly composed of $11 \mathrm{~S}$ globulins (37\%), $2 \mathrm{~S}$ albumins $(35 \%)$, and small amount of prolamins (0.5$7.0 \%)[8,9]$.

Among all these technical functionalities of proteins, gelation is considered as one of the most important attributes, as it play important roles in textures of various food products such as yoghurt, sausage, meat analogue and tofu-like products. As globular proteins, QPI can form gels under heat treatment. Upon heating above the denaturation temperature of the QPI $\left(\sim 75^{\circ} \mathrm{C}\right)$, compact protein structures start to unfold and hydrophobic amino acid groups that were buried inside of the proteins are exposed, leading to protein aggregations through hydrophobic interactions [10]. When the protein concentration is greater than the minimum protein concentration required for the gelation, these protein aggregates can further grow to large clusters 
and finally formed a three dimensional interconnected network structure [11]. Previous studies suggested that the QPI gelation kinetics and the resultant gel characteristics such as gel strength are strongly related to extraction procedure, temperature, $\mathrm{pH}$, and ionic strength $[10,12]$. For example, a previous study indicated that protein fractions of lower molecular weight became more prominent as the extraction $\mathrm{pH}$ increased from 8 to 11 . Thus, only QPI obtained from extractions at $\mathrm{pH} 8$ and $\mathrm{pH} 9$ can from self-supporting gels [12].

Compared to animal and dairy derived proteins, most of plant protein gels exhibited a weak gel strength, as they are mostly formed by non-covalent interactions [13]. This limit their applications in food and pharmaceutical products and thus improvement of gel strength is needed. Incorporation of polysaccharides into protein gels is one of most commonly used strategies to enhance the protein gel strength and microstructure [14]. Depending on the nature of proteins and polysaccharides as well as $\mathrm{pH}$, temperature, and ionic strength, interactions between proteins and polysaccharides can be either through electrostatic attraction or intermolecular repulsion [15]. The latter may lead to thermodynamic incompatibility or depletion-flocculation phenomena, resulting in a phase separation [16]. Both types of interactions have been reported to lead to the improvement of gelation properties [17, 18]. For example, Zhao, Chen, Hemar and Cui [19] found that the mechanical properties of $\mathrm{Ca}_{2} \mathrm{SO}_{4}$ induced soy protein isolate gels were significantly improved after additions of $0.5 \%(\mathrm{w} / \mathrm{v})$ of konjac gum, gellan gum, and curdlan gum.

Guar gum (GG) is a neutral polysaccharide obtained from seeds of the plant Cyamopsis tetragonoloba, a member of the Leguminosae family [20, 21]. In terms of structure, GG has a linear backbone of mannose units with branched chains constituting galactose units [21]. The galactose to mannose ratio is $1: 2$, therefore the degree of branching is $\sim 50 \%$. The higher hydrogen bonding and hydration property of guar gum are due to a large degree of branching [22]. Locust bean gum (LBG) has a galactose to mannose ratio of 1:4 and also belongs to the galactomannan family [23, 24]. Like GG, LBG is also a non-ionic neutral gum [24]. Xanthan gum (XG) is an anionic polysaccharide generated by bacterial of the genus Xanthomonas [25]. XG produces a viscous solution and has been used extensively as a stabiliser or thickener in many food applications [26]. XG comprises a linear cellulose backbone substituted with a trisaccharide side chain containing glucose, mannose, and gluconate as well as acetyl and pyruvate groups [27]. Owing to different molecular structural characteristics (e.g. molecular weight and degree of branching) of GG, LGB, and XG; their hydration and selfassociation behaviour in water as well as interactions with proteins could be different. Thus, the incorporations of different polysaccharides may result in QPI gels with different rheological properties, water-holding capacities, and microstructural characteristics.

Most studies on properties of polysaccharide-protein mixtures to fabricate composite gels are focused on animal or dairy-derived proteins, and such studies on emerging plant proteins are limited. Therefore, the aim of this work is to study the gelation behaviour and microstructural characteristics of heat-induced QPI gels in the presence of three polysaccharides-GG, LBG, and XG at three concentrations. This study will provide useful information for developing QPI gel based food products.

\section{Materials and Methods}

\section{Materials}

Quinoa seeds (Chenopodium quinoa) were kindly provided from Kiwi Quinoa, and they were locally grown and processed in Taihape, New Zealand. Chemicals including $\mathrm{HCl}$, $\mathrm{NaOH}, \mathrm{NaCl}$, petroleum ether, and sodium azide were purchased from Sigma-Aldrich (USA) and were of analytical grade. Polysaccharides including guar gum (GG, purity: 92\%, Mw: $\sim 1000 \mathrm{kDa}$ ), locust bean gum (LBG, purity: 92\%, Mw: 1200 kDa), and xanthan gum (XG, purity:90\%, Mw: 2000 kDa) were obtained from DuPont Danisco, Inc. (Singapore). Milli- $Q$ water was used for all the sample preparation.

\section{Preparation of quinoa protein isolate (QPI)}

The quinoa protein isolate (QPI) was obtained according to our previous studies $[28,29]$. The raw quinoa seeds were pulverised using a coffee grinder (Coffee and spice grinder, Breville, New Zealand) and sieved through a $500 \mu \mathrm{m}$ sieve (Endecotts, UK). The ground quinoa flour was soaked and stirred continuously in petroleum ether for $24 \mathrm{~h}$ in a ratio of $1: 5(\mathrm{w} / \mathrm{w})$ and then dried in a fume hood at $\sim 20^{\circ} \mathrm{C}$ for $15 \mathrm{~h}$. Defatted quinoa flour was then soaked in $0.5 \mathrm{M} \mathrm{NaCl}$ solution containing $0.02 \mathrm{wt} \%$ sodium azide, with a weight ratio of quinoa flour to $\mathrm{NaCl}$ solution 1:10. Under magnetic stirring, the $\mathrm{pH}$ of the suspension was then adjusted to 8 with $1 \mathrm{M} \mathrm{NaOH}$ and kept stirring overnight to maximize the protein extraction at $20^{\circ} \mathrm{C}$. pH was regularly check and adjusted when necessary. Thereafter, the suspension was centrifuged at $10000 \times g$ at $20^{\circ} \mathrm{C}$ (Sigma, 6-16KS with rotor 12356 , Germany) for $15 \mathrm{~min}$, and the supernatant was collected. The residual fat layer on the surface of the supernatant was carefully removed by a wooden spatula. This centrifugation step was repeated twice until the supernatant exhibited low turbidity. After that, the $\mathrm{pH}$ of the supernatant was adjusted to 4.5 using $1 \mathrm{M} \mathrm{HCl}$, before centrifugating at $10000 \times g$ at $20{ }^{\circ} \mathrm{C}$ for 15 min to recover the precipitated protein. The 
collected protein pellets were thoroughly washed with Milli$\mathrm{Q}$ water and centrifuged under the same conditions for three times until the electrical conductivity became lower than 40 $\mu \mathrm{S} / \mathrm{cm}$. The pellets were then redispersed to Milli- $Q$ water containing $0.02 \mathrm{wt} \%$ sodium azide at $\mathrm{pH} 7$ under magnetically stirring overnight. Finally, The QPI solution was frozen at $-20{ }^{\circ} \mathrm{C}$ for $48 \mathrm{~h}$, before being lyophilised using a freeze dryer (Labconco, Model 7753034, USA) for 5 days. A pestle and mortar was used to grind the dried sample to fine powder. The obtained QPI powder was stored in sealed plastic containers and kept in a desiccator avoiding sunlight for further use. The proximate analyses of QPI were conducted at the Nutrition Laboratory of Massey University (School of Food and Advanced Technology, Palmerston North, New Zealand). The protein, fat, ash, and moisture contents of the QPI powder were $91.6 \%, 2.9 \%, 2.8 \%$, and $2.7 \%$, respectively.

\section{Sample preparations}

The QPI stock solution (12.5wt $\%$ ) was prepared by dispersing the QPI powder in Milli- $Q$ water containing $0.02 \mathrm{wt} \%$ sodium azide under stirring for $24 \mathrm{~h}$ to allow the protein hydration and solubilisation. To prevent microorganisms growth, sodium azide wad added. Polysaccharides stock solution (1.0 wt $\%$ ) was prepared by dispersing the GG, LBG, or XG powder in Milli- $Q$ water containing $0.02 \mathrm{wt} \%$ sodium azide under stirring for $\sim 5 \mathrm{~h}$. Polysaccharides stock solution was left overnight at $20{ }^{\circ} \mathrm{C}$ to enable full hydration. The gum-QPI mixtures were prepared by mixing an appropriate amount of QPI stock solution, different polysaccharide stock solution, $1 \mathrm{M} \mathrm{NaCl}$ solution, and Milli- $Q$ water containing $0.02 \%$ sodium azide. The ionic strength of all samples was fixed at $50 \mathrm{mM} \mathrm{NaCl}$ in order to promote gelation of QPIgum composites by diminishing electrostatic repulsions between protein molecules [28]. All dispersions were gently mixed under magnetically stirring for $2 \mathrm{~h}$ at $20^{\circ} \mathrm{C}$, and the $\mathrm{pH}$ was regularly checked and adjusted to 7 using $1 \mathrm{M} \mathrm{HCl}$ or $1 \mathrm{M} \mathrm{NaOH}$.

\section{Confocal Laser Scanning Microscopy (CLSM)}

CLSM was used to observe the microstructures of various QPI dispersions and gels. In brief, a few drops of $1 \%$ fast green dye was added and mixed with QPI dispersions and then vortex mixed for 15 seconds at $20{ }^{\circ} \mathrm{C}$. Thereafter, aliquots of each sample were carefully transferred into a glass slide with cavity and covered with a glass coverslip. In the case of QPI gels, to prevent water evaporation [30], nail polish was used to seal the coverslip edges before transferring into an oven (Labserve, Australia) pre-set at $85{ }^{\circ} \mathrm{C}$ for 30 $\mathrm{min}$. The samples were observed at a wavelength of $630 \mathrm{~nm}$ under a confocal laser scanning microscope (Leica TCS SP5,
Leica Microsystems) attached with a $100 \times$ oil immersion objective lens. The Image J software (version $1.51 \mathrm{k}, \mathrm{NIH}$, USA) was used to process all images.

\section{Rheological characterisations}

Dynamic rheological measurements were conducted using a DHR-3 stress-controlled rheometer (TA instruments, USA) equipped with a stainless steel plate-plate geometry (diameter: $40 \mathrm{~mm}$, gap: $1 \mathrm{~mm}$ ). Aliquots of QPI dispersions were carefully transferred onto the bottom plate using a plastic dropper. The samples were then carefully trimmed before adding the low viscosity mineral oil (M5904, Sigma Aldrich, USA) around the edge of the sample to prevent evaporation during the rheological measurements. The following rheological protocols were conducted in a sequence: firstly, a temperature sweep was performed during which the sample was heated from 25 to $85^{\circ} \mathrm{C}$ at $1{ }^{\circ} \mathrm{C} / \mathrm{min}$, maintained at 85 ${ }^{\circ} \mathrm{C}$ for $30 \mathrm{~min}$, before decreasing to $25^{\circ} \mathrm{C}$ at $1{ }^{\circ} \mathrm{C} / \mathrm{min}$. During this step, the gel formation was monitored by recording evolution of storage modulus $G^{\prime}$ and loss modulus $G^{\prime \prime}$ at a fixed frequency of $1 \mathrm{~Hz}$ and a fixed strain of $1 \%$. Secondly, to investigate viscoelasticity of heat induced gels, a frequency sweep was carried out at a constant strain $1 \%$ while the frequency was changed from 0.01 to $30 \mathrm{~Hz}$. Finally, large deformation rheological properties of samples were characterised by a strain sweep test, and the frequency was fixed at $1 \mathrm{~Hz}$, while the strain amplitude was varied from $0.1 \%$ to $1000 \%$. All the frequency and strain sweep measurements were performed at $25{ }^{\circ} \mathrm{C}$.

\section{Water holding capacity (WHC)}

The water holding capacity was determined according to Zhao, Chen, Hemar and Cui [19] with slight modifications. QPI dispersions (10 wt $\%, 2 \mathrm{~g}$ each) were transferred to Eppendorf tubes and heated in a water bath pre-set at $85^{\circ} \mathrm{C}$ for $30 \mathrm{~min}$ to form gels. The gels were left on bench to allow temperature to equilibrate to room temperature $\left(\sim 20^{\circ} \mathrm{C}\right)$. Thereafter, the gels formed in the tubes were centrifuged at $4000 \times \mathrm{g}$ for $20 \mathrm{~min}$ at $20^{\circ} \mathrm{C}$ (Heraeus Pico 17 centrifuge, Thermo scientific, USA). WHC (\%) was calculated as the weight ratios of the wate left in gels after centrifugation to that of the water in the original samples before centrifugation.

\section{Statistical analysis}

All measurements were conducted twice with duplicated samples, and the data was represented as the mean \pm standard deviation. Analysis of variance (ANOVA) was conducted with the SPSS software (version 21.0, IBM, NY, 


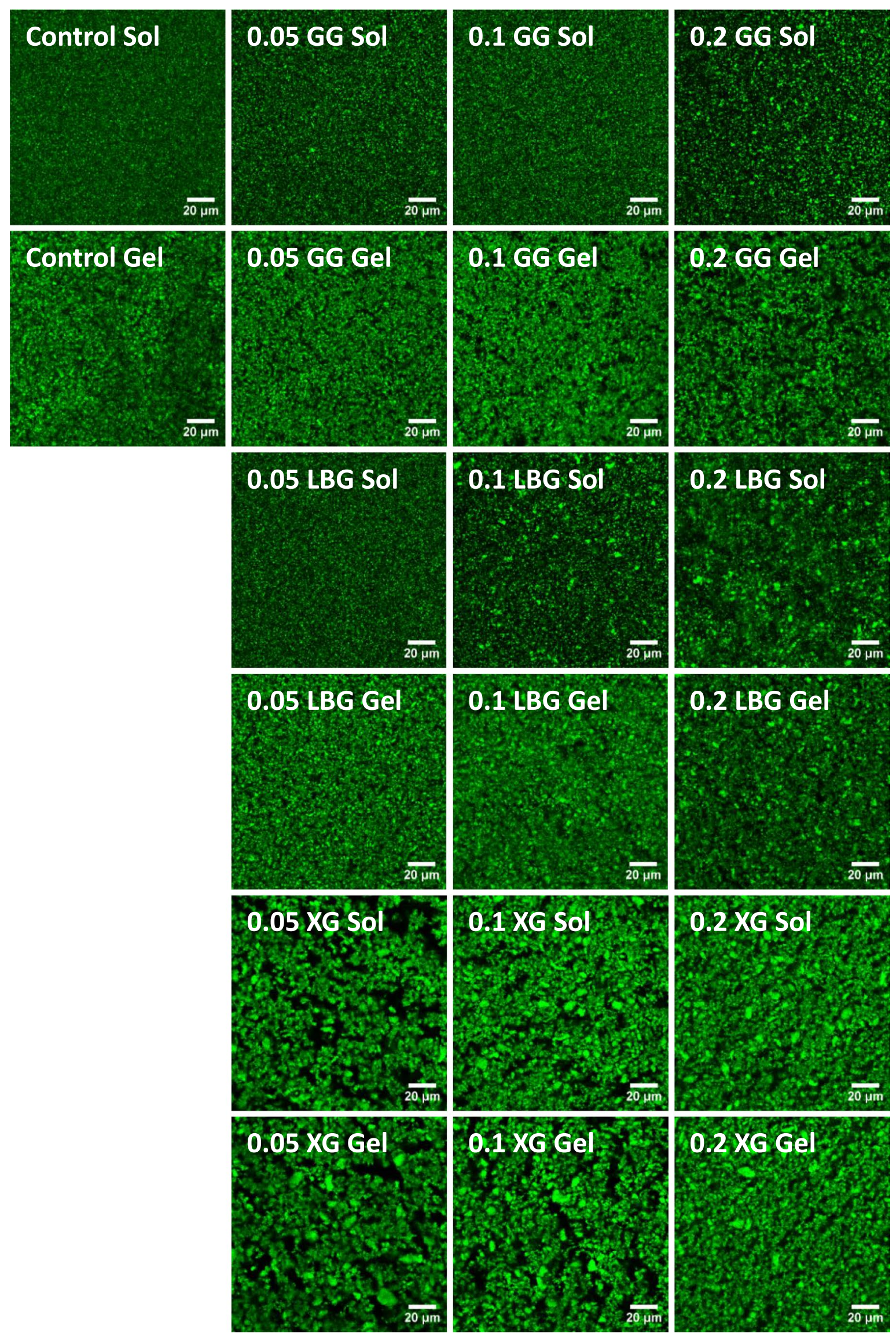


४Fig. 1 Confocal laser scanning micrographs of $10 \mathrm{wt} \%$ QPI dispersions and gels in the absence and presence of guar gum, locust bean gum, and xanthan gum at $0.05 \mathrm{wt} \%, 0.1 \mathrm{wt} \%$, and $0.2 \mathrm{wt} \%$. The gels were formed by heating at $85^{\circ} \mathrm{C}$ for $30 \mathrm{~min}$.

USA) using the Least Significant Difference (LSD) test at the significance level $P<0.05$.

\section{Results and Discussion}

\section{Microstructural characteristics revealed by CLSM}

Confocal laser scanning microscopy (CLSM) has been commonly employed to characterise the microstructural characteristics of protein-polysaccharide mixtures [14]. Confocal micrographs of QPI dispersions containing different polysaccharides including guar gum (GG), locust bean gum (LBG), and xanthan gum (XG) at various concentrations before (sol) and after (gel) heat treatment are shown in Fig. 1. Protein stained with fast green appear as green, while the polysaccharides rich zones and voids in between the protein particles appeared in dark. For all QPI samples, interconnected protein network structures were formed after heat treatment, which resulted in gel characteristics as agreed with previous studies on heat induced QPI gels [10, 12, 28]. Incorporation of all the polysaccharides made structures heterogenous by forming large protein particles, and these heterogeneities being more prominent in heat treated samples. Heat treatment results in protein denaturation, which enhances the exposing of hydrophobic groups, thus promoting proteinprotein interactions through hydrophobic interactions [13]. Further, at all polysaccharide concentrations, XG additions induced formation of largest protein aggregates and microphase separation followed by that of LBG and GG. Similar observations have been made in heat-induced WPI gel in the presence of XG [18], NaCl-induced cold set gels of soy protein isolate containing LBG and GG [31], and acid induced soy milk gels adding with GG, LBG, and XG [32]. This was attributed to the fact that the mixtures of proteins and polysaccharides are incompatible because of their low mixing entropy [14].

QPI carry a negative charge at $\mathrm{pH} 7$, as the isoelectric point of QPI is 4.5-5.0 [9]. The addition of both neutral (GG and LBG) and anionic polysaccharides (XG) could induce various degree of incompatibility [14]. For examples, rheological properties and structural characteristics of soy protein gel at $\mathrm{pH} \sim 7$ containing GG and LBG $(0.1-0.3 \%)$ were evaluated by Brito-Oliveira, Cavini, Ferreira, Moraes and Pinho [31]. The authors also observed that the microstructure became more heterogenous with the addition of GG and LBG, while the latter induced more predominant phase separation. The authors explained this by the strong self-association of LBG due to its less branched structure (branching 25\%) than GG (branching 50\%) as above discussion. Likewise, Bertrand and Turgeon [18] found that the incorporation of XG into WPI gel at $\mathrm{pH} 6.0$ and 6.5 destroyed the connectivity of the protein network because of incompatibility of these polymers at these gelling conditions. It can be seen from Fig. 1 that the protein network structure became more heterogenous with the increase in polysaccharides concentrations. This observation is supported by a number of previous studies [31, 32]. In addition, it has been suggested that within a certain range, the gel strength increased with the increase in the degree of incompatibility or micro-phase separation $[18,33]$. The incorporation of polysaccharides may also enhance network via depletion flocculation or steric exclusion mechanisms [16]. Proteinsolvent interactions are considerably suppressed by incorporation of polysaccharide thus promoting the protein-protein interactions [34].

It is worth noting that even at $\mathrm{pH}>\mathrm{pI}$ where QPI carry a negative charge, the electrostatic attraction can also occur between anionic gums (like XG) and cationic amino acids located in some protein patches of QPI [14]. While for neutral gums like GG and LGB, they can only form hydrogen bonding with proteins. The electrostatic association is likely to occur between QPI and XG, and these interactions could give rise to a more compact and denser structures and great gel strength particularly at higher concentration of XG $(0.2 \%)$. A similar observation has been found in bigeye snapper surimi containing another anionic polysaccharide-gellan gum [35]. The great gel strength and a higher degree of micro-phase separation in QPI-XG gel could be also ascribed to a high molecular weight of XG. It has been suggested that the polysaccharide with a high molecular weight or longer chain such as XG could occupy much larger hydrodynamic volume, facilitating them to interact with other polymers [36]. Ozel, Cikrikci, Aydin and Oztop [37] found that owing to a higher molecular weight, XG generated more compact structures than tragacanth gum in the WPI gels at $\mathrm{pH}$ 7. In another study working on galactomannan-soy protein gels at $\mathrm{pH} 7$, Monteiro and Lopes-daSilva [38] found that the incorporation of the galactomannan with larger molecular weight gave rise to more extensive phase separation, lower gelation temperature, and greater gel stiffness. The authors explained this by the enhancement of protein-protein interactions (via. hydrophobic interactions and hydrogen bonding) and polysaccharide-polysaccharide interactions (via. molecular entanglement) in each phase.

\section{Effect of polysaccharides incorporation on the rheological properties during gelation}

Viscoelastic properties of the QPI dispersion in the presence of GG, LBG, and XG at various concentrations 
during heat-induced gelation were monitored by small dynamic oscillatory deformation measurement. Storage modulus $\left(G^{\prime}\right)$ and loss modulus $\left(G^{\prime \prime}\right)$ as a function of temperature are recorded and presented in Fig. 2. The $G^{\prime}$ and $G^{\prime \prime}$ indicate the elastic and viscous component of QPI gels, respectively. Qualitatively, all the samples displayed a similar behaviour and both $G^{\prime}$ and $G^{\prime \prime}$ increased throughout the entire course of heat treatment (temperature increase, maintaining, and decrease). The onset of
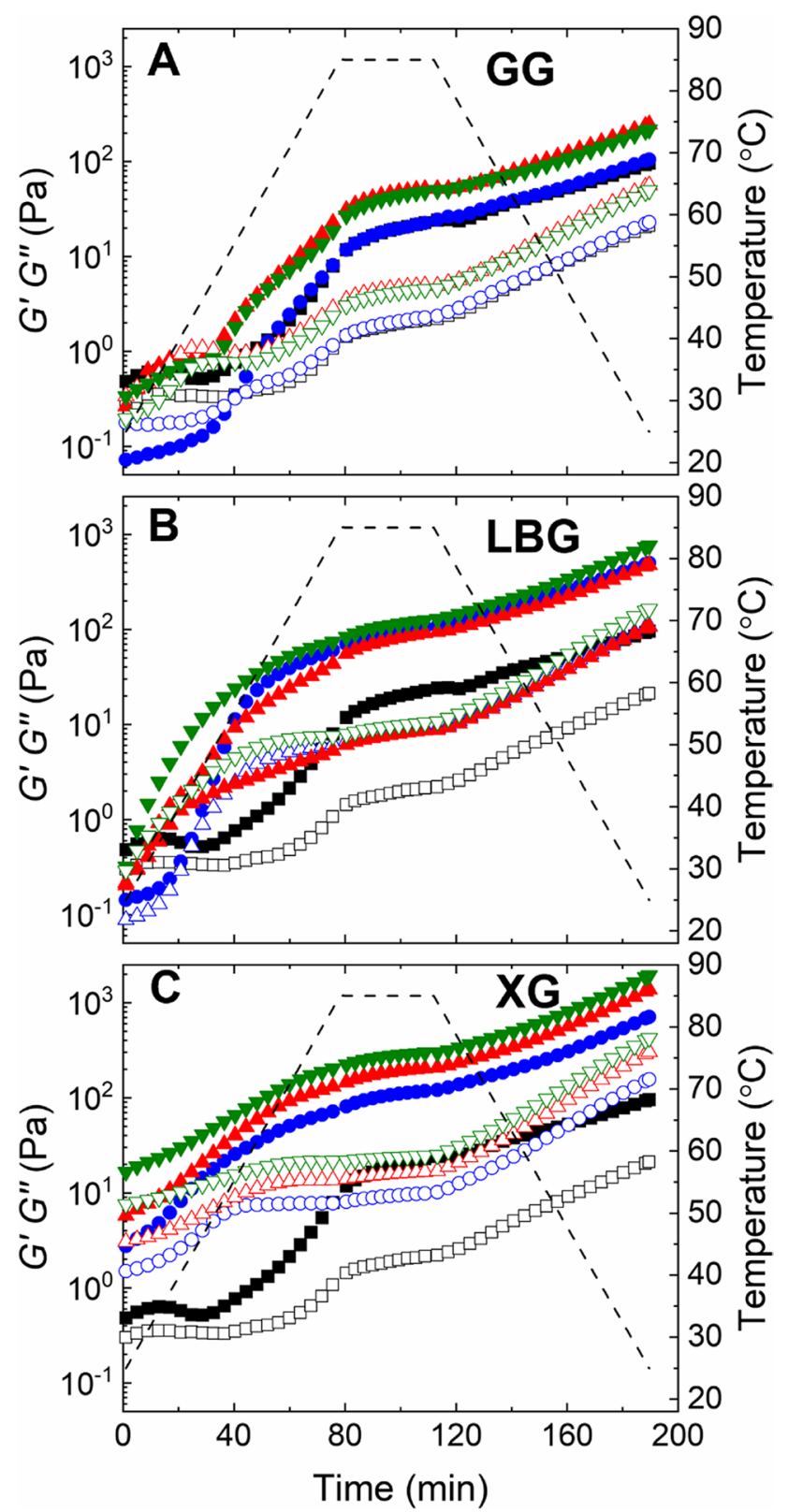

Fig. 2 The evolution of $G^{\prime}$ (solid symbols) and $G^{\prime \prime}$ (open symbols) for $10 \mathrm{wt} \%$ QPI dispersions during heat-induced gelation in the absence and presence of guar gum (A), locust bean gum (B), and xanthan gum (C). Gums concentrations: $0 \mathrm{wt} \%(\boldsymbol{\square}), 0.05 \mathrm{wt} \%(\bullet), 0.1 \mathrm{wt} \%(\mathbf{\triangle})$, and $0.2 \mathrm{wt} \%(\nabla))$. gelation, $\mathrm{T}_{\text {gel }}$, when $G^{\prime}$ reached $1 \mathrm{~Pa}$ [39] during the temperature ramp stage are shown in Fig. 3. Note that at time zero, $G^{\prime}$ values of the QPI dispersion in the presence of $X G$ are higher than $1 \mathrm{~Pa}$, thus the $\mathrm{T}_{\text {gel }}$ for those samples cannot be determined using this method. This also indicates that the sol-gel transition occurred rapidly in the presence of XG at room temperature, which could be due to a high water binding and gel forming capacity of $\mathrm{XG}$ at $25^{\circ} \mathrm{C}$ (Fig. S1). Generally, for both GG and LBG, the $\mathrm{T}_{\text {gel }}$ was considerably decreased with the increase in concentrations of polysaccharides. At the same concentration, $\mathrm{LBG}$ exhibited a larger reduction in $\mathrm{T}_{\text {gel }}$ than that of GG. For example, compared to the control, incorporation of $0.2 \mathrm{wt} \%$ of GG and LBG into QPI dispersion resulted in a decrease of $\mathrm{T}_{\text {gel }}$ from $\sim 70{ }^{\circ} \mathrm{C}$ to $\sim 52{ }^{\circ} \mathrm{C}$ and $\sim 32$ ${ }^{\circ} \mathrm{C}$, respectively. Similar gelation behaviours have been found in previous gelation studies of various plant proteins with adding polysaccharides. Pang, Luo, Li, Zhang and Liu [32] found that $\mathrm{T}_{\text {gel }}$ of acid induced soymilk gels decreased from $\sim 13 \mathrm{~min}$ to $\sim 8.5 \mathrm{~min}, \sim 8.0 \mathrm{~min}$, and $\sim 7.6$ min, respectively in the presence of $0.10 \% \mathrm{GG}, \mathrm{LBG}$, and $\mathrm{XG}$, respectively. In another study working on $\mathrm{Ca}_{2} \mathrm{SO}_{4}$ induced soy protein isolate gels, Zhao, Chen, Hemar and Cui [19] indicated that gelation occurred earlier when added with 0.3 and $0.5 \%$ of konjac gum, gellan gum, and curdlan gum. It has been suggested that the incorporation of polysaccharides can improve interactions among proteins, thus facilitating gelling of protein-gum dispersions at lower temperatures [34]. Owing to strong water binding capacities of polysaccharides, protein-protein interactions are promoted and is accompanied with a decrease of protein-water association, leading to an earlier aggregation

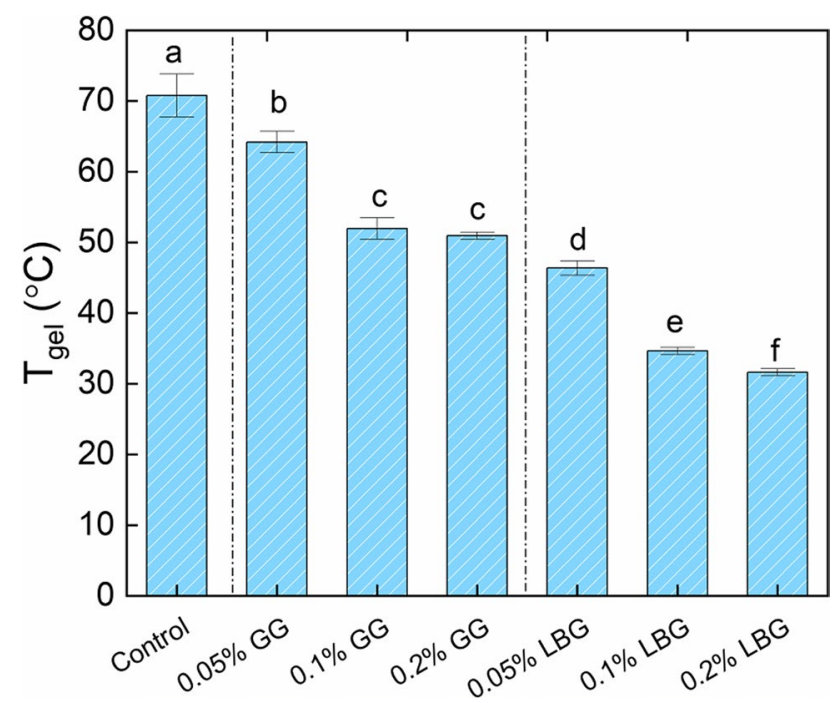

Fig. 3 The gelation temperature $\mathrm{T}_{\text {gel }}$ of the QPI gels containing guar gum and locust bean gum at $0 \mathrm{wt} \%, 0.05 \mathrm{wt} \%, 0.1 \mathrm{wt} \%$, and $0.2 \mathrm{wt} \%$. Different letters above columns indicate significant differences. 
and gelation in QPI-gum composites [40]. Molecular interactions between polysaccharides and proteins may affect rheological properties and microstructural characteristics of the final composite gels. Due to a charge screening effect of $\mathrm{NaCl}$ and neutral nature of $\mathrm{GG}$ and LBG, inclusions of polysaccharides did not induce significant changes on surface charges of the QPI as revealed by zeta-potential measurements (results not shown). Nevertheless, further studies are required to probe non-covalent interactions between polysaccharides and proteins (if any) using Fourier-transform infrared spectroscopy (FTIR) and/or nuclear magnetic resonance (NMR). In addition, differential scanning calorimetry (DSC) can be used to examine the effect of polysaccharides addition on unfolding/denaturation of the QPI.

Despite both GG and LBG are galactomannans, LBG exhibited a less degree of branching $(\sim 25 \%)$ than that of GG ( 50\%) [14]. Therefore, LBG demonstrated a higher capacity to self-associate and showed more incompatible with proteins, thereby promoting the protein-protein interactions to a greater extent [31]. This could give rise to the greater $T_{\text {gel }}$ reduction in the presence of LBG than GG. Alternatively, lower branching of LBG might improve the integration in the QPI protein network, resulting in a more prominent increase of $G^{\prime}$ with the increase in temperature (Fig. 2). Future studies such as staining proteins and polysaccharides with different dyes in CLSM experiments and/or directly probe protein-polysaccharide interactions at a molecular level using FTIR/DSC/NMR may help to understand the underlying mechanism.

At the latter stage of temperature ramping and holding at $85^{\circ} \mathrm{C}$, both $G^{\prime}$ and $G^{\prime \prime}$ progressively increased but at a much lower rate. This indicated that the unfolding and denaturation of protein molecules occurred progressively and hydrophobic amino acids group that were buried inside of the protein were increasingly exposed to induce protein aggregation. When the protein concentration beyond the critical concentration for gelation, protein aggregates are further grown into large clusters and eventually to form an interconnected three dimensional network structure. Similar trend of $G^{\prime}$ and $G^{\prime \prime}$ versus heating temperature have been found in previous reports on rheological studies of heat-induced QPI gels [10, 28]. The heat-induced gelation is one of the most common gelation route for globular proteins and similar gelation behaviours have been also found in previous studies on whey protein isolates (WPI) [41], pea protein isolates [13], and faba bean protein isolates [42]. When the temperature is decreased, all the QPI gels showed an increase in $G^{\prime}$ and $G^{\prime \prime}$, which is likely due to the formation or enhancement of covalent and non-covalent bonds such as hydrogen bond and disulfide bond among proteins, as well as the interactions between protein-polysaccharides $[19,36]$.

\section{Effect of polysaccharides incorporation on the rheological properties after gelation}

At the end of temperature sweep measurements, the small and large deformation rheological properties of QPI gels in the presence of different gums at various concentrations were characterised by the frequency sweep and strain sweep measurement and the results are demonstrated in Fig. 4. A constant strain of $1 \%$ was applied in all the frequency sweep measurements to ensure the strain is well within the Linear Viscoelastic Region (LVER) region. $G^{\prime}$ and $G^{\prime \prime}$ as a function of frequency for all QPI gels are shown in Fig. 4A, B, and C. For all the QPI samples, $G^{\prime}$ is greater than $G^{\prime \prime}$ over the whole frequency range and both are slightly dependent of frequency, demonstrating typical characteristics of weak gels [13, 30]. To allow better comparison of gel strength among different QPI gels, the complex modulus $G^{*}$ at $1 \mathrm{~Hz}$ which takes into account the contribution from both $G^{\prime}$ and $G^{\prime \prime}$ at this frequency are presented in Fig. 4A. Compared to control, the gel strength is increased in the presence of all the polysaccharides and greater gel strength is achieved at higher polysaccharide concentrations. Further, the effect of gel strengthening is strongly related to the type of polysaccharides. Incorporating XG resulted in highest gel strength at all gum concentrations followed by LBG and GG. For instance, in the presence of $0.2 \% \mathrm{XG}, 0.2 \% \mathrm{LBG}$, or $0.2 \%$ $\mathrm{GG}$, the value of $G^{*}$ increased $\sim 15, \sim 7$, and $\sim 2$ folds than the control sample, respectively. The greatest gel strength in the QPI gels containing XG could be attributed to the largest protein aggregates and prominent phase separation as revealed by CLSM. The improvement of gel strength of protein gels upon addition of polysaccharides have been found in many previous studies [31, 43-45]. The degree of enhancement in gel strength are affected by intrinsic factors such as the nature, conformation, and concentration of the protein and polysaccharide and by extrinsic factors including heating temperature, $\mathrm{pH}$, and ionic strength [14].

In the case of large deformation rheology, the dependence of $G^{\prime}$ and $G^{\prime \prime}$ with applied strain for all QPI samples are presented in Fig. 4D, E, and F. At lower strains, $G^{\prime}$ and $G^{\prime \prime}$ are independent of the applied strain until reaching a critical strain value, which is defined as the LVER region. Above this point, $G^{\prime}$ and $G^{\prime \prime}$ begin to deviate from plateau and $G^{\prime}$ decreased more steeply than $G^{\prime \prime}$ with further increase in the applied strain. Eventually, crossover of $G^{\prime}$ and $G^{\prime \prime}$ occurs, and beyond this point $G^{\prime \prime}$ is greater than $G^{\prime}$, indicating network structure of gels is broken down and start to flow. The stress at this crossover point $\left(G^{\prime}=G^{\prime \prime}\right)$ is defined as breaking or yield stress. To better examine the large deformation rheological properties differences among the various QPI gels containing different polysaccharides, the breaking stress (BS) for the different samples is summarized in Fig. 5B. Generally, the breaking stress increased with the increase in 
Fig. 4 The dependence of frequency $(\mathrm{A}, \mathrm{B}, \mathrm{C})$ and strain amplitude (D, E, F) on $G^{\prime}$ (solid symbols) and $G^{\prime \prime}$ (open symbols) for QPI dispersions at $10 \mathrm{wt} \%$ with various concentrations of polysaccharides incorporation measured at 25 ${ }^{\circ} \mathrm{C}$. Gums concentrations: 0 wt $\%(\boldsymbol{\square}), 0.05 \mathrm{wt} \%(\bullet), 0.1 \mathrm{wt} \%$ $(\triangle)$, and $0.2 \mathrm{wt} \%(\nabla))$.

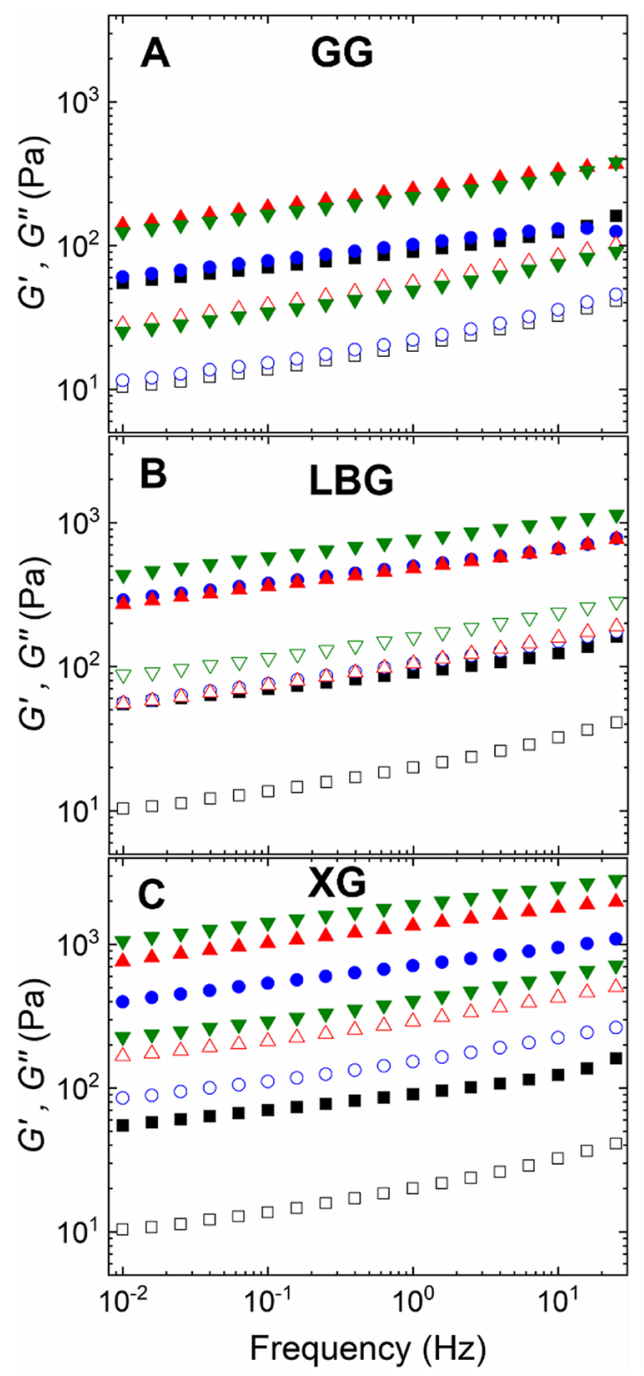

polysaccharide concentration in a polysaccharide dependent manner, following the nearly same trend as $G^{*}(1 \mathrm{~Hz})$. For example, QPI gels containing XG exhibited greatest value of $\mathrm{BS}$, indicating these gels are more resistant toward structural rearrangement and yield when subjected to large oscillatory deformation. In summary, the small and large deformation rheological behaviour correlate well with the microstructural characteristics (e.g. size of protein aggregates, extent of microstructural separation, and structural inhomogeneities) as revealed by CLSM.

\section{Impact of polysaccharides addition on the WHC}

The WHC (\%) of QPI gels in the presence and absence of various polysaccharides at different concentrations is shown in Fig. 5C. Incorporation of GG did not induce changes in WHC, while the additions of $0.2 \mathrm{wt} \% \mathrm{LBG}$ and $\mathrm{XG}$ (at all concentrations) markedly improved the WHC. For example, the WHC increased from $\sim 50 \%$ to $\sim 90 \%$ after adding $0.2 \mathrm{wt} \% \mathrm{XG}$ to the QPI gel. WHC is closely related to compactness of structural arrangements within the network and a high WHC typically resulted in a greater gel strength [14, 43]. This is also observed in the present study that the compact structure induced by XG addition particularly at 0.1 and $0.2 \mathrm{wt} \%$ (Fig. 1) could entrap water tightly and could not easily be disrupted by centrifugation force, which was responsible for the high WHC and stiffer gels. It could be attributed to the fact that QPI-XG co-polymers have a strong water absorption and swelling capacity and prevent the water channels formation between proteins, leading to a compact network of QPI proteins and great WHC [43, 46]. Similar findings were also found in previous studies that the incorporation of $0.05 \% \mathrm{XG}$ significantly improved the WHC and gel strength of soy milk gels [32].

\section{Conclusions}

GG, LBG, and XG have been extensively used in food products for modifying textures and improving stabilities. In the current study, the impact of their incorporations on 

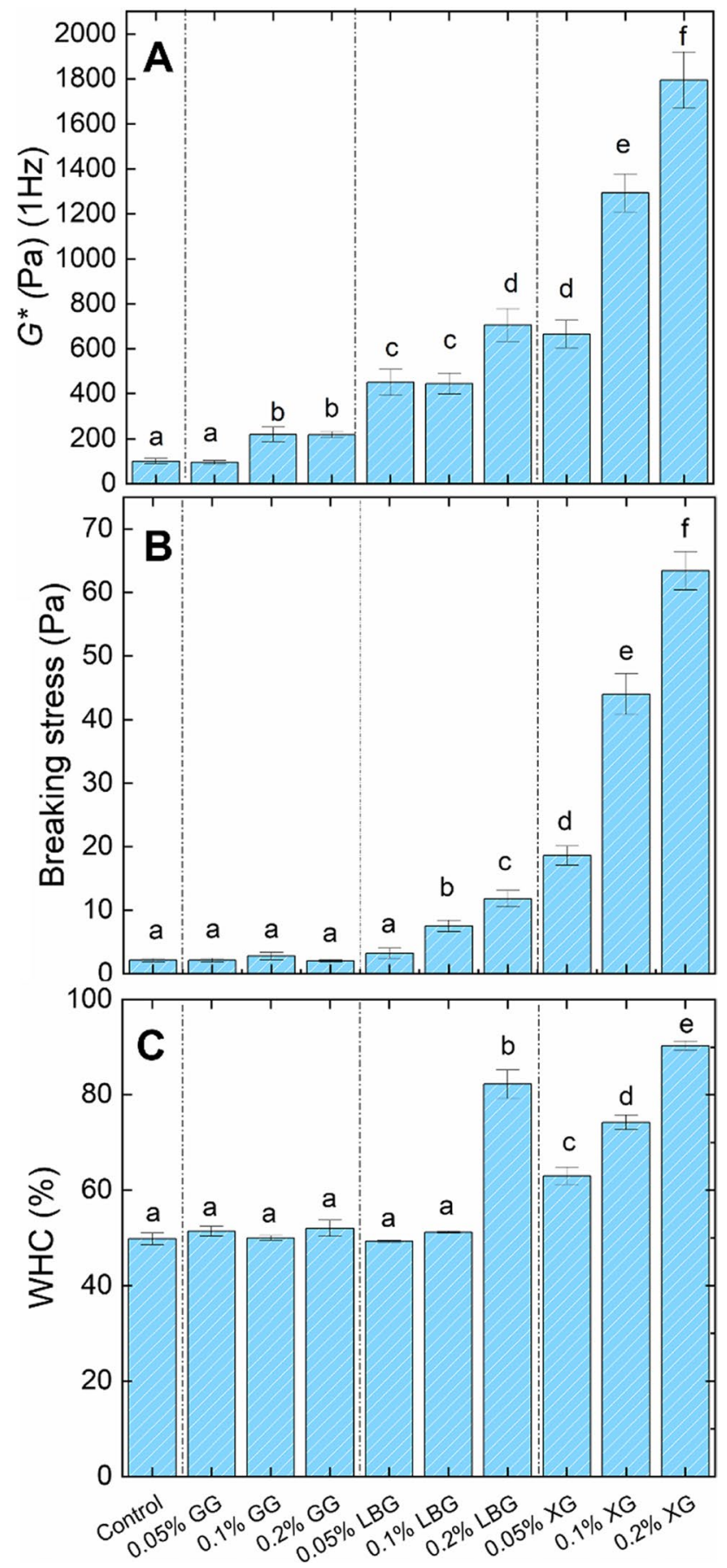

Fig. 5 Complex modulus $G^{*}(1 \mathrm{~Hz})(\mathrm{A})$, breaking stress (B), and water holding capacity $(\mathrm{C})$ of the QPI gels containing guar gum, locust bean gum, and xanthan gum at $0 \mathrm{wt} \%, 0.05 \mathrm{wt} \%, 0.1 \mathrm{wt} \%$, and $0.2 \mathrm{wt} \%$. Different letters above columns indicate a significant difference.

the rheological properties and microstructural characteristics of heat-induced QPI gels were evaluated. The gel strength and network structures were improved by addition of abovementioned polysaccharides, as evidenced by the increase in $G^{\prime}$ and $G^{\prime \prime}$ during and after gelation. The gel strength and microstructural features are strongly dependent on the polysaccharide used, which might be attributed to different extents of demixing and/or interactions between the QPI and polysaccharides. Owing to a high molecular weight and anionic nature, $\mathrm{XG}$ is most effective in improving the gel strength followed by LBG and GG. Since LBG has a lower degree of branching than GG, it has a higher capacity to self-associate or it is more compatible with the QPI, resulting in stronger gels. In addition, the gel formation is accelerated by incorporating polysaccharides, as reflected in the decrease in the gelation temperature, $\mathrm{T}_{\text {gel. }}$. For all polysaccharides, the complex modulus, breaking stress, WHC were progressively improved with increasing incorporation of polysaccharides. Various extents of polysaccharide-protein demixing were observed by CLSM with QPI gels containing XG displaying the most prominent micro-phase separation. The gel structures became more compact and denser with increasing addition of XG. Nevertheless, further studies are needed to understand molecular interactions between polysaccharides and the QPI. The finding obtained from this study could be useful for development of food products containing QPI gels with desirable gel stiffness, textures, and microstructures. Finally, different polysaccharides and food ingredients (e.g. small saccharides and salts) might co-exist in QPI gel based food products. Therefore, it may be worth investigating the impact of other polysaccharides (e.g. kappa and iota-carrageenan and alginate) $[47,48]$, gelatine [49], small sugars (e.g. sucrose) $[50,51]$, and salts (e.g. $\mathrm{NaCl}$ and $\left.\mathrm{CaCl}_{2}\right)[31,52]$ on the rheological properties and microstructural characteristics of QPI gels.

Supplementary Information The online version contains supplementary material available at https://doi.org/10.1007/s11483-022-09720-3.

Acknowledgements Zhi Yang would like to thank the financial support by the College of Sciences Massey University Research Fund (MURF) for Early Career Researchers. We appreciated the support from the kiwi Quinoa company for kindly providing the quinoa seeds used in the current work. We would also like to thank Dr. Yanyu He from the Manawatu Microscopy Imaging Centre (MMIC) for providing support during the confocal laser scanning microscopy experiments.

Authors' contributions Shubham Patole: Investigation, Formal analysis, Writing-original draft, Lirong Cheng: Investigation, Formal analysis, Writing-review \& editing, Zhi Yang: Conceptualization, Supervision, Writing-original draft, Writing-review \& editing, Funding acquisition. All authors read and approved the manuscript.

Funding Open Access funding enabled and organized by CAUL and its Member Institutions. 


\section{Declarations}

Conflict of interest The authors declare that there is no competing financial interests in this work.

Open Access This article is licensed under a Creative Commons Attribution 4.0 International License, which permits use, sharing, adaptation, distribution and reproduction in any medium or format, as long as you give appropriate credit to the original author(s) and the source, provide a link to the Creative Commons licence, and indicate if changes were made. The images or other third party material in this article are included in the article's Creative Commons licence, unless indicated otherwise in a credit line to the material. If material is not included in the article's Creative Commons licence and your intended use is not permitted by statutory regulation or exceeds the permitted use, you will need to obtain permission directly from the copyright holder. To view a copy of this licence, visit http://creativecommons.org/licenses/by/4.0/.

\section{References}

1. L. Day, J.A. Cakebread, S.M. Loveday, Trends in Food Science \& Technology 119, 428-442 (2022)

2. M. Niva, A. Vainio and P. Jallinoja, in Vegetarian and plant-based diets in health and disease prevention (Elsevier, 2017), pp. 157-171.

3. A.G.A. Sá, Y.M.F. Moreno, B.A.M. Carciofi, Trends in Food Science \& Technology 97, 170-184 (2020)

4. L. Le Roux, R. Chacon, D. Dupont, R. Jeantet, A. Deglaire, F. Nau, Food Research International 130, 108917 (2020)

5. S. Sethi, S.K. Tyagi, R.K. Anurag, Journal of Food Science and Technology 53(9), 3408-3423 (2016)

6. O.E. Mäkinen, V. Wanhalinna, E. Zannini, E.K. Arendt, Critical Reviews in Food Science and Nutrition 56(3), 339-349 (2016)

7. S. Dakhili, L. Abdolalizadeh, S.M. Hosseini, S. Shojaee-Aliabadi, L. Mirmoghtadaie, Food Chemistry 299, 125161 (2019)

8. L. E. Abugoch, N. Romero, C. A. Tapia, J. Silva and M. Rivera, Journal of Agricultural and Food chemistry 56 (12), 4745-4750 (2008).

9. Y. Shen, X. Tang, Y. Li, Food Chemistry 339, 127823 (2021)

10. E. Kaspchak, M. A. S. d. Oliveira, F. F. Simas, et al., Food Chemistry 232, 263-271 (2017).

11. M. Chen, C. Zhao, Chassenieux, T. Nicolai, Food Hydrocolloids 70, 88-95 (2017)

12. G.A. Ruiz, W. Xiao, M. van Boekel, M. Minor, M. Stieger, Food Chemistry 209, 203-210 (2016)

13. X.D. Sun, S.D. Arntfield, Food Hydrocolloids 28(2), 325-332 (2012)

14. M.C. Cortez-Trejo, M. Gaytán-Martínez, M.L. Reyes-Vega, S. Mendoza, Trends in Food Science \& Technology 116, 303-317 (2021)

15. A. Ye, International Journal of Food science \& Technology 43 (3), 406-415 (2008)

16. Hemar, M. Tamehana, P. A. Munro and H. Singh, Food Hydrocolloids 15 (4), 565-574 (2001).

17. C.M. Bryant, D.J. McClements, Food Hydrocolloids 14(4), 383$390(2000)$

18. M.-E. Bertrand, S.L. Turgeon, Food Hydrocolloids 21(2), 159$166(2007)$

19. H. Zhao, J. Chen, Y. Hemar, B. Cui, Food Chemistry 310, 125983 (2020)

20. A. George, P.A. Shah, P.S. Shrivastav, European Polymer Journal 112, 722-735 (2019)

21. D. Mudgil, S. Barak and B. S. Khatkar, Journal of Food science and Technology 51 (3), 409-418 (2014).
22. N. Thombare, U. Jha, S. Mishra, M. Siddiqui, International Journal of Biological Macromolecules 88, 361-372 (2016)

23. G. Sébastien, B. Christophe, A. Mario, L. Pascal, P. Michel, R. Aurore, Carbohydrate Polymers 108, 159-168 (2014)

24. V.D. Prajapati, G.K. Jani, N.G. Moradiya, N.P. Randeria, B.J. Nagar, Carbohydrate Polymers 94(2), 814-821 (2013)

25. F. Garcia-Ochoa, V. Santos, J. Casas, E. Gómez, Biotechnology Advances 18(7), 549-579 (2000)

26. S.V. Niknezhad, M.A. Asadollahi, A. Zamani, D. Biria, M. Doostmohammadi, Food Science and Biotechnology 24(2), 453-460 (2015)

27. A. Kumar, K.M. Rao, S.S. Han, Carbohydrate Polymers 180, 128-144 (2018)

28. Z. Yang, L. de Campo, E.P. Gilbert, et al., Food Hydrocolloids 124, 107350 (2022)

29. R. Zhang, L. Cheng, L. Luo, Y. Hemar, Z. Yang, Colloids and Surfaces A: Physicochemical and Engineering Aspects 631, 127688 (2021)

30. Y. Wang, B. Eastwood, Z. Yang, et al., Food Hydrocolloids 96, 161-170 (2019)

31. T. C. Brito-Oliveira, A. C. M. Cavini, L. S. Ferreira, I. C. F. Moraes and S. C. Pinho, Food Structure, 100158 (2020).

32. Z. Pang, Y. Luo, B. Li, M. Zhang, X. Liu, Food Hydrocolloids 101, $105558(2020)$

33. Y.L. Tan, A. Ye, H. Singh, Y. Hemar, Journal of Texture Studies 38(3), 404-422 (2007)

34. Y.C. Tseng, Y.L. Xiong, Journal of Food Engineering 90(4), 511-516 (2009)

35. T. Petcharat, S. Benjakul, Food Hydrocolloids 77, 746-753 (2018)

36. X. Yang, A. Li, D. Li, Y. Guo, L. Sun, Trends in Food Science \& Technology 109, 197-210 (2021)

37. B. Ozel, S. Cikrikci, O. Aydin, M.H. Oztop, Food Hydrocolloids 71, 35-46 (2017)

38. S. R. Monteiro, S. Rebelo, O. A. B. da Cruz e Silva and J. A. Lopes-da-Silva, Food Hydrocolloids 33 (2), 349-360 (2013).

39. J.A. Lucey, H. Singh, Food Research International 30(7), 529-542 (1997)

40. L. Luo, R. Zhang, J. Palmer, Y. Hemar, Z. Yang, ACS Food Science \& Technology 1(11), 2144-2151 (2021)

41. C. F. Diedericks, V. Stolten, V. A. Jideani, P. Venema and E. van der Linden, Food Hydrocolloids, 106702 (2021).

42. M. Langton, S. Ehsanzamir, S. Karkehabadi, X. Feng, M. Johansson, D.P. Johansson, Food Hydrocolloids 103, 105622 (2020)

43. X. Zhuang, X. Jiang, H. Zhou, et al., Carbohydrate Polymers 229, 115449 (2020)

44. I. M. Geremias-Andrade, N. P. D. B. G. Souki, I. C. F. Moraes and S. C. Pinho, LWT 86, 166-173 (2017).

45. X. Zhuang, B. Cheng, W. Kang, X. Xu, Carbohydrate Polymers 82(2), 524-527 (2010)

46. Y. Hemar, S. Lebreton, M. Xu, L. Day, Food Hydrocolloids 25(4), 668-676 (2011)

47. Z. Ren, Z. Li, Z. Chen, et al., Food Hydrocolloids 114, 106562 (2021)

48. L.C. Sow, J.M.N. Chong, Q.X. Liao, H. Yang, Journal of Food Engineering 239, 92-103 (2018)

49. M. Yin, D. Yang, S. Lai, H. Yang, LWT 147, 111643 (2021)

50. D. Yang, S. Gao, H. Yang, Food Hydrocolloids 99, 105317 (2020)

51. Z. Yang, H. Yang, H. Yang, Food Hydrocolloids 75, 164-173 (2018)

52. Y. Zhou, H. Yang, Food Chemistry 277, 327-335 (2019)

Publisher's Note Springer Nature remains neutral with regard to jurisdictional claims in published maps and institutional affiliations. 